\title{
Dinamica da alteração do indice técnico do arremesso jump e sua relação com a aptidão neuromuscular de potencia no basquete feminino
}

\section{Dynamics of changes in jump shot performance and its relationship with increased neuromuscular power in women's basketball}

\author{
Laline Oliveira da Silva
}

\begin{abstract}
RESUMO: O objetivo do presente estudo foi analisar a dinâmica das alteraçōes do índice técnico do arremesso jump ( ITA) através de ajustes cinemáticos e a correlaçấo destes com aptidão neuromuscular de potencia (ANMP) no basquetebol feminino .Para isso foram selecionadas dezesseis atletas profissionais (idade média de 22,49 $\pm 5,2$ anos; massa corporal 71,42 $\pm 11,47 \mathrm{~kg}$; estatura $180,12 \pm 10,25 \mathrm{~cm}$.) pertencentes a uma mesma equipe participante da Liga Brasileira de Basquete Feminino (LBF). As atletas foram divididas em dois grupos, onde cada um recebeu seu protocolo de treinamento de potencia muscular diferente no decorrer de oito semanas, tendo seu ITA e sua ANMP avaliados em seis etapas durante e após a intervenção nas variáveis antropométricas, de potencia muscular de membros superiores e inferiores e no ITA. Os resultados apresentaram um aumento significativo na ANMP $(\mathrm{p}<0,05)$ do grupo que recebeu um protocolo de treinamento de cargas seletivas em relação ao grupo que recebeu um protocolo de treinamento de cargas concentradas. Essa diferença significativa refletiu-se no grau de correlação $(\mathrm{p}<0,001)$ das variáveis do grupo seletivo em relação ao grupo de cargas concentradas alterando consequentemente de modo distinto o ajuste cinemático do ITA entre os grupos.Concluímos que o grupo que recebeu um treinamento com cargas seletivas obteve uma melhor eficácia no índice técnico do arremesso e isso esteve diretamente relacionado com a melhora da aptidão neuromuscular de potencia desse grupo.

Palavras-chaves: índice técnico do arremesso jump; aptidão neuromuscular de potencia; basquetebol feminino.
\end{abstract}

ABSTRACT: The aim of this study was to analyze the dynamics of changes in the jump shot indicator (ITA) by means of kinematics adjustments and the correlation of these adjustments with increased neuromuscular power (ANMP) in women's basketball. Therefore, sixteen professional athletes (mean age of $22.49 \pm 5.2$ years; body weight $71.42 \pm 11.47 \mathrm{~kg}$; height $180.12 \pm 10.25 \mathrm{~cm}$ ) of a team in the Brazilian League of Women's Basketball were selected. They were divided into two groups, and each group received its own training protocol on muscle power (endurance) in the course of eight weeks, and their ITA and ANMP were evaluated in six steps during and after the intervention in the anthropometric variables, muscle power of upper and lower limbs and ITA. The results showed a significant increase in ANMP $(\mathrm{p}<0.05)$ in the group that received a training program of selective load compared to the group that received a training program of concentrated load. This significant difference reflected in the degree of correlation $(\mathrm{p}<0.001)$ of the variables of the selective load group compared to the concentrated load group, thus promoting different kinematics adjustments of ITA in the groups. We concluded that the group that received the training protocol of selective load was more efficient regarding jump shot performance and this was directly related to increased neuromuscular power in this group.

Key-words: jump shot performance; neuromuscular power; women's basketball.

\section{Introduçáo}

Entre os tipos de arremesso utilizados em uma partida de basquetebol o arremesso jump apresenta o maior índice de frequência, sendo responsável atualmente por $41 \%$ do total de pontos de uma partida. (Rojas e cols.2000). Esse destaque deve-se segundo Okazaki e cols.2006; Okazaki, 2004a principalmente ao fator do arremesso jump proporcionar maior vantagem como precisão, velocidade de execução, proteção contra a marcaçáo e execuçáo a diversas distâncias da cesta. Os estudos cinemáticos referentes ao arremesso jump na área de biomecânica estão focados em vários segmentos, mas todos apresentam em comum a analise das variáveis que determinam as características de vôo da bola que são a velocidade, o ângulo e a altura de liberação da mesma. (Okazacki, 2004

Doutoranda Universidad de León.

Email: lalineoliveira28@yahoo.com.br a). A excelente altura, ângulo e velocidade de liberação da bola determinam a eficácia e precisão dessa habilidade motora, (Rodacki, 2001).

O arremesso jump é um gesto técnico caracterizado por se manifestar inúmeras vezes e exigindo esforços máximos explosivos em um trabalho curto e intensivo, alternados com intervalos curtos de trabalho pouco intensivo, mantendo o alto nível de precisão espacial e de movimento. (Bompa, 2002). Há um consenso na literatura de que para o desenvolvimento da potência muscular uma estratégia eficiente parece ser o treinamento de força. Kyrolainen, Avela, Mcbride, Koskinen, Andersen, Sipila, Takala e Komi, 2005; Harris, Stone, O Bryant, Proulx e Johnson, 2000 citado por Lamas e cols., 2008. Todavia a eficiência desta estratégia está relacionado a distribuição da carga utilizada, seu volume, intensidade e sistema de treinamento. (Baker, 2001; Verkhoshansky, 2001).

O sistema de treinamento das cargas concentradas é um 
sistema caracterizado por quatro aspectos: (a) a individualização das cargas de treinamento, organismo; (b) a concentraçáo de cargas de trabalho de uma mesma orientação em períodos de curta duração; (c) a tendência a um desenvolvimento consecutivo de capacidades e objetivos, utilizando o efeito residual de cargas trabalhadas anteriormente; (d) o incremento do trabalho específico de treinamento.( Verkhoshansky,2001; Manso, Navarro e Caballero, 1996; Borin, 2007). O sistema de cargas seletivas tem como objetivo principal o aperfeiçoamento da capacidade de velocidade e em uma segunda instância a de força. Nesse sistema a velocidade de movimento é de fundamental importância na evolução da performance, por isso indicando prioriza-se o desenvolvimento do sistema nervoso muscular, sucedendo o trabalho anterior cujo conteúdo predominante deve ser da resistência especial, com crescente utilização dos meios e métodos direcionados para o aperfeiçoamento da força, velocidade e particularidades técnicas e táticas. (Moreira, 2005).

\section{Metodologiga}

\section{Amostra}

Foram recrutadas 16 jogadoras profissionais de basquete, que disputaram ( LBF -2010/2011) (média de idade foi de 22,49 $\pm 5,2$ anos; massa corporal 71,42 $\pm 11,47 \mathrm{~kg}$; estatura $180,12 \pm$ $10,25 \mathrm{~cm}$. )As atletas foram divididas em dois grupos de oito jogadoras baseadas na sua posição de jogo, com cada grupo composto por duas armadoras, quatro alas e quatro pivôs. Durante a pré-temporada partir da quarta semana cada grupo recebeu um protocolo especifico de treinamento para a potencia muscular. O grupo que recebeu o protocolo baseado no conceito de cargas concentradas ficou denominado como G1; e o grupo que recebeu um protocolo de treinamento baseado no conceito de cargas seletivas de G2. As atletas assinaram voluntariamente o Termo de Consentimento Livre e Esclarecido. O estudo foi aprovado pelo Comitê de Ética em Pesquisa da Universidad de León.

\section{Protocolo experimental}

As atletas foram submetidas à avaliação de potencia muscular de membros superiores e inferiores; antropométrica e técnica do arremesso jump na pré-temporada e no decorrer das 12 semanas da fase competitiva da LBF 2010/2011. As avaliaçóes foram realizadas em uma quadra oficial de basquetebol, de acordo com as regras da Federaçáo Internacional de Basquete (FIBA). Todas as atletas receberem na sua apresentação um protocolo de treinamento de força máxima promovendo dessa forma uma similaridade na aptidáo de força para ambos os grupos antes da intervençáo. $\mathrm{O}$ primeiro teste (T1) foi realizado na quarta semana após a apresentação da equipe durante a pré- temporada. O segundo teste (T2), foi realizado na décima segunda semana de treinamento após cada grupo receber um protocolo especifico de desenvolvimento da potencia. O terceiro teste (T3) se efetuou no inicio da fase competitiva na semana do primeiro jogo. O quarto teste (T4) foi efetuado na quarta semana após o primeiro jogo, na fase regular da competiçáo. O quinto teste (T5) foi realizado na oitava semana após o inicio da competição, na fase de returno da mesma. O sexto teste (T6) foi realizado na décima segunda semana de competição ao final da fase regular

- Avaliação da composição corporal: Mensurou-se o peso corporal, a estatura, e as dobras cutâneas tricipital, suprailíaca e coxa. Calcularam-se o percentual de gordura e a massa magra através do protocolo de Guedes, 1994.

- Avaliação da aptidão neuromuscular de potencia: A potencia muscular de membros superiores foi mensurada por meio dáo teste de medicine ball de $2 \mathrm{~kg}$ e a potencia muscular de membros inferiores pelo tapete de contato . A medida de potencia muscular de membros inferiores foi realizada através dos testes de saltos verticais : contra movimento (CMJ), squat jump (SJ) e Abalakov (ABK).

- Avaliação do ITA : Foi composta pelas variáveis técnicas temporais, posicionais e de velocidade conforme determina o protocolo de Rojas ,2000; e pela variável técnica de precisão de deslocamento da bola, conforme determina o protocolo de Okazaki e cols. 2006b.

\section{Tratamento estatistico}

Foi realizado a média e o desvio padrão para calcular a estatística descritiva das diferentes variáveis analisadas. Adotou-se o teste de Kolmogorov-Smirnov para uma amostra a modo de determinar a normalidade. $\mathrm{O}$ teste $\mathrm{t}$ para amostras independentes foi utilizado para descartar a existência de diferenças significativas entre os grupos no inicio de aplicação dos protocolos de treinamento. Para efetuar a analise comparativa da evolução da diferentes variáveis entre ambos os grupos ao longo da aplicação dos protocolos foi utilizado uma ANOVA de dois fatores com medidas repetidas (grupo $\mathrm{x}$ tempo). O procedimento estatístico de referencia foi a Lambda de Wilks e o teste post-hoc de Tukey para localizar diferenças significativas quando constatadas. O teste de coeficiente de correlação de Pearson foi utilizado para verificar a correlação entre as variáveis. Foi adotado um valor de significância de $\mathrm{p}<0,05$.

\section{Resultados}

Os resultados apresentaram uma diferença significativa ANMP $(p=0,03)$ no grupo que recebeu um protocolo de treinamento de cargas seletivas em relação ao outro grupo e uma correlação significativa com as variáveis $\operatorname{ABK}(\mathrm{p}=0,001)$; 
$\mathrm{SJ}(\mathrm{p}=0,04)$ variáveis técnicas posicionais $(\mathrm{p}=0,02)$, temporais $(\mathrm{p}=0,01)$, velocidade $(\mathrm{p}=0,001)$, precisão $(\mathrm{p}=0,01), \mathrm{MB}$ $(\mathrm{p}=0,04), \mathrm{PC}(\mathrm{p}=0,02)$ e PG $(\mathrm{p}=0,03)$; o grupo que recebeu um protocolo de treinamento de cargas concentradas não apresentou diferenças significativas na ANMP em relação ao outro grupo , mas apresentou correlação significativa nas variáveis técnicas de velocidade $(\mathrm{p}=0,001)$, temporais $(\mathrm{p}=0,001$ ), $\operatorname{ABK}(\mathrm{p}=0,01), \mathrm{CMJ}(\mathrm{p}=0,01), \mathrm{MM}(\mathrm{p}=0,03), \mathrm{MB}(\mathrm{p}=0,04)$, precisão $(\mathrm{p}=0,03)$.

Tabela 01: Correlações entre as variáveis no G1. *A correlação é significativa ao nível 0,05 (bilateral).** A correlação é significativa ao nível 0,01 (bilateral).

\begin{tabular}{lccccccccccc}
\hline Variável & Peso & $\% \mathrm{G}$ & $\% \mathrm{MM}$ & $\mathrm{MB}$ & $\mathrm{CMJ}$ & $\mathrm{SJ}$ & $\mathrm{ABK}$ & $\begin{array}{c}\text { Variáveis } \\
\text { posicionais }\end{array}$ & $\begin{array}{c}\text { Variáveis } \\
\text { temporais }\end{array}$ & $\begin{array}{c}\text { Variáveis de } \\
\text { velocidade }\end{array}$ & $\begin{array}{c}\text { Variáveis de } \\
\text { precisão }\end{array}$ \\
\hline C. Pearson & 0,54 & 0,14 & $0,31^{*}$ & $0,27^{*}$ & $0,63^{* *}$ & 0,52 & $0,77^{* *}$ & 0,43 & $0,79^{* *}$ & $0,82^{* *}$ & $0,32^{*}$ \\
Sig.(Bilateral) & 0,06 & 0,08 & 0,03 & 0,04 & 0,01 & 0,06 & 0,01 & 0,07 & 0,001 & 0,001 & 0,03 \\
$\mathrm{~N}$ & 08 & 08 & 08 & 08 & 08 & 08 & 08 & 08 & 08 & 08 & 08 \\
\hline
\end{tabular}

Tabela 02: Correlações entre as variáveis no G2. Correlações entre as variáveis no G1. *A correlação é significativa ao nível 0,05 (bilateral).** A correlação é significativa ao nível 0,01 (bilateral).

\begin{tabular}{lcccccccccccc}
\hline Variável & Peso & $\% \mathrm{G}$ & $\% \mathrm{MM}$ & $\mathrm{MB}$ & $\mathrm{CMJ}$ & $\mathrm{SJ}$ & $\mathrm{ABK}$ & $\begin{array}{c}\text { Variáveis } \\
\text { posicionais }\end{array}$ & $\begin{array}{c}\text { Variáveis } \\
\text { temporais }\end{array}$ & $\begin{array}{c}\text { Variáveis de } \\
\text { velocidade }\end{array}$ & $\begin{array}{c}\text { Variáveis de } \\
\text { precisão }\end{array}$ \\
C. Pearson & $0,31^{*}$ & $0,35^{*}$ & 0,12 & $0,19^{*}$ & 0,22 & $0,34^{*}$ & $0,81^{* *}$ & $0,63^{* *}$ & $0,71^{* *}$ & $0,84^{* *}$ & $0,72^{* *}$ \\
Sig.(Bilateral) & 0,02 & 0,03 & 0,09 & 0,04 & 0,06 & 0,04 & 0,001 & 0,02 & 0,01 & 0,001 & 0,01 \\
$\mathrm{~N}$ & 08 & 08 & 08 & 08 & 08 & 08 & 08 & 08 & 08 & 08 & 08 \\
\hline
\end{tabular}

\section{Discussão}

Os dados demonstram um aumento significativo de potencia muscular ao longo da intervenção aplicada em ambos os grupos, porem com uma dinâmica diferente entre eles o que podemos considerar estar de acordo com a literatura afirmando que os aspectos da bioadaptação estão diretamente relacionados com o tipo de modelo de treinamento adotado. (Bompa, 2002; Manso, 1998).

$\mathrm{Na} \mathrm{PMI}$ os resultados apresentaram um aumento significativo do grupo que treinou com cargas seletivas em relação ao grupo que treinou com cargas concentradas. Esse aumento estendeu-se para a correlação entre as variáveis de ANMP e o ITA, demonstrando um numero maior de variáveis correlacionadas e com o nível de significância estatística maior. Esses resultados estão de acordo com os trabalhos de Moreira e cols., 2005 e Cetolin e cols. 2010 que demonstram nesse método de treinamento que tem como principal objetivo o aperfeiçoamento da velocidade durante a fase competitiva da temporada esportiva. O grupo de cargas concentradas apresentou um numero inferior de variáveis correlacionadas, porém manteve seu ajuste cinemático de forma crescente durante a temporada muito mais linear que o grupo que treinou com cargas seletivas. Esse fator pode estar relacionado com o efeito duradouro pós treinamento onde devido ao grande volume e intensidade executados de uma maneira mais concentrada promove uma reestruturaçóes morfológicas mais eficaz. (Moreira, 2005, Manso e cols. 1998.)

Na variável técnica temporal que no grupo de cargas concentradas apresentou um nível de correlação excelente, e no grupo de cargas seletivas apresentou um nível de correlaçáo muito boa, nota-se nos seus indicadores de velocidade de deslocamento e de tempo total um aumento significativo nos indicadores de tempo de vôo que conforme Rojas e cols. 2000, esse ajuste temporal faz com que a atleta alcance uma maior potencia de pico na elevação do CG e por consequência tenha melhores condiçóes temporais de um ajuste cinemático para a execuçáo do lançamento. Outra variável de destaque que obteve um excelente nível de correlação entre ambos os grupos foi a variável técnica de velocidade que correlacionada com a potencia muscular reflete em uma redução no tempo de deslocamento horizontal do CG durante todo o movimento com uma tendência maior de diminuição na fase inicial do deslocamento, o que nessa modalidade esportiva é de grande importância a modo de conseguir ter mais êxito na saída da marcação do jogador adversário. (Rojas, 2000).Todas estas variáveis técnicas conforme já descrito acima apresentam uma correlação significativa com o nível de aptidáo neuro- 
muscular de potencia que segundo Verkoshansky, 2001; se dá devido a fatores como a maximização do recrutamento das unidades motoras; aumento da frequência de recrutamento; desenvolvimento da coordenação intermuscular que tem sua dinâmica de desenvolvimento intimamente relacionado com o tipo de treinamento aplicado. No presente estudo essa dinâmica ocorreu de acordo com a método de distribuição dessa carga especifica de treinamento foi aplicada para cada grupo, com impacto de seus resultados relacionados com a variável técnicas responsáveis pelo êxito no arremesso jump.

\section{Referencias bibliograficas}

Bompa, T. (2002). Periodização - Teoria e Metodologia do Treinamento. São Paulo. Editora Phorte.

Borin, J.P; y Gomes, A.C; Leite, G.( 2007). Sporting preparation: aspects of load training control in collective games. Revista da Educação Física UEM, 18 (1), 21-27.

Cetolin, T. y Foza, V. (2010). Periodizaçấo no futsal: descrição da utilização da metodologia de treinamento baseada nas cargas seletivas. Brazilian Journal of Biomotricity, 4 (1), 24-31.

Guedes, D. P. y Guedes, J. E. (1998). Controle do peso corporal: composição corporal, atividade física e nutrição. Londrina. Midiograf.

Harris, G.R.; Stone, M.H.; O’ Bryant, H.S.; Proulx, C.M. y Johnson, R.L.(2000). Short-term performance effects of high power, high force, or combined weight-training methods. Journal of Strength and Conditioning Research, Champaign, 14(1), 14-20.

Hudson, J. L. (1985). Prediction of basketball skill using biomechanical variables, Research Quarterly for Exercise and Sport, 56 (1), 115 - 121.

Kellis, E, Arambatzi, F. y Papadopoulos, C.(2005). Effects of load on ground reaction force and lower limb kinematics during concentric squats. Journal of Sports Sciences, 23(10), 1045-55.

Kyrolainen, H.; Avela, J.; Mcbride, J.M.; Koskinen, S.; Andersen, J.L.; Sipila, S.; Takala, T.E. y Komi, P.V. (2005). Effects of power training on muscle structure and neuromuscular performance. Scandinavian Journal of Medicine and Science in Sports .15 (1), 58-64.

Lamas, L., Drezner, R.; Tricoli, V. y Ugrinowitsch, C. (2008). Efeito de dois métodos de treinamento no desenvolvimento da força máxima e da potência muscular de membros inferiores. Revista Brasileira de Educação Física. São Paulo, 22 (3), 31-38.

Manso, J.G; Navarro, M. y Caballero, J.A. (1996). Bases teoricas del entrenamiento deportivo. Madrid. Gymnos.

Miller, S. y Bartlett, R. M. (1996). The relationship between basketball shooting kinematics, distance and playing position. Journal of Sports Sciences .14, 243- 253

Moreira, A., Okano, A., Hidecki, A. y Oliveira, P.R. (2005).Change for speed and strength measures with selective loads system in basketball players during a mesocycle. Revista Brasileira de Ciências do Movimento. $13(3), 7-16$

\section{Conclusão}

A partir desse estudo concluímos que a aptidão neuromuscular de potencia está significativamente relacionada com os indicadores técnicos do arremesso jump, e sua contribuição ao longo da temporada possui uma ligação direta ao tipo de protocolo de treinamento que está submetido o atleta. O grupo que recebeu o protocolo de cargas seletivas obteve uma melhor eficácia no seu ajuste cinemático, porém seus ajustes apresentaram um tempo mais curto de êxito ao longo da temporada em relaçáo ao grupo de treinamento de cargas concentradas.
Okazaki, V.H.A., Rodacki, A.L.F., Sarraf, T.A.; Dezan, V.H. y Okazaki, F. H. (2004). Diagnóstico da especificidade técnica dos jogadores de basquetebol. Revista Brasileira de Ciência e Movimento, 12 (4), 17-24.

Okazaki, V.H.A., Oliveira, G.O., Ferreira Júnior, R. y Rodacki, A.L.F. (2006 a). Coordenação do arremesso jump no basquetebol de crianças. Fédération Internationale D'éducation Physique, 76 (2) ,523-526.

Okazaki, V.H.A.; Rodacki, A.L.F.; Okazaki, F.H.A. (2006 b). O efeito do aumento da distância na coordenaçáo do arremesso de jump no basquetebol e a relação velocidade-precisão. Lecturas: Educación Física y Deportes. Acesso em 20/05/2012 http://www.efdportes.com

Okazaki, V.H.A.; Rodacki, A.L.F.; Okazaki, F.H.A. (2006c). O Arremesso de jump no basquetebol. Lecturas: Educación Física y Deportes. Acesso em 20/05/2012 http://www.efdportes.com

Rodacki, A.L.F., Fowler, N.E., Bennet, S. (2001).The effect of postural variations in movement co-ordination during plyometric rebound exercises. Journal of Applied Biomechanics, 17, 14-27.

Rojas, F. J., Cepero, M., Onä, A., Gutiérrez. (2000). Kinematic adjustments in the basketball jump shot against an opponent. Ergonomics, 43 (10), 1651- 1660 .

(2000). Diferencias biomecánicas entre jugadores principiantes y del alto rendimiento en el lanzamiento en salto en baloncesto. Biomecánica, 8 (1), 3-14.

Salgado., Sedano, S, Trigueros, A., Izquierdo, J.M. y Cuadrado, G.(2009) Anthropometric profile of Spanish female basketball players. Analysis by level and by playing position. International Journal Sport Science, 5 (15), 21-29.

Toumi, H., Best, T.M., Martin, A. y Poumarat, G. (2004). Muscle plasticity after weight and combined (weight + jump) training. Medicine and Science in Sports and Exercise,

36 (9), 1580-1588.

Vazquez, A.; Gayo A. , Pita H.; , Fernandez, C. (2011). Design and appli cation of a multidimensional battery of performance indicators for evaluating competitive performance in top-level football. International Journal of Sport Science. 7 (23), 103-112.

Verkhoshansky, Y. V.(2001). Treinamento esportivo: teoria e metodologia. Porto Alegre, Artmed. 